\title{
Design and Realization a Versatile Performance Test Platform for Motorcycle Anti-lock Braking System
}

\author{
Shu-Heng Guo*, Chun-Hung Wu, Kuo-Shen Chen \\ Department of Mechanical Engineering, National Cheng-Kung University, \\ No.1, Daxue Rd., East Dist., Tainan City 701, Taiwan \\ *Corresponding Author: n16064373@mail.ncku.edu.tw
}

\begin{abstract}
Anti-lock braking system (ABS) is an active safety system for vehicle, it operates by preventing the wheels from locking up during braking. According to a lot of research, the effectiveness of $\mathrm{ABS}$ in reducing crashes is significant. To design the ABS system, establishing an ABS test bench, which is capable for analyzing the ABS braking performance, the individual ABS components performance, and the whole ABS durability is necessary. In this work, we upgraded our original hydraulic control unit performance test bench to satisfy the above mentioned goals by further enhancing the function in this module test bench and further designing and realizing an ABS dynamic braking performance test bench. The module test bench can be used for evaluating the performance of whole $\mathrm{ABS}$ product quickly and precisely. In addition, the durability of the hydraulic control units and the control logic of the ABS can be performed and analyzed by the aid of a virtual speed signal circuit built in this test bench. On the other hand, the designed ABS dynamic performance test bench is aimed for testing the braking performance of $\mathrm{ABS}$ by using the flywheel design to simulate the field test environment. Finally, These two test benches are eventually integrated to form a versatile test platform. Through the effort, in the future, it is possible to perform effective performance assessment on commercial ABS systems and to develop new control strategies based on this test platform.
\end{abstract}

Keywords: anti-lock braking system, hydraulic control unit, ABS performance test, ABS braking performance test

\section{Introduction}

Anti-lock braking systems(ABS) is an active safety braking system for preventing slipping and have been implanted in many kinds of vehicles. ABS systems can prevent wheels from locking up and reduce braking distance. Meanwhile, it can help vehicles maintain steering ability. Over the past few decades, more and more motorcycles and cars have installed ABS. Many countries and companies have listed them as standard equipment. In Taiwan, the government announced that 126c.c. motorcycle or above must equip ABS since 2019. For reacting to the rapid demand in the market, it is important to establish the ability in design and realize ABS systems. Among the many tasks involved, one key issue is to design and realize a versatile $A B S$ performance and durability testing facility for quickly evaluating the performances of both the self-designed and the comparator products for accelerating the development cycles.

There are many ABS testing systems designed for academia research and industrial testing. For academia research, people usually designed their test systems mainly for studying the controller design. For example, Shih et al. ${ }^{(2)}$ designed a sliding mode PWM control to perform ABS braking control. Obviously, such test benches cannot be used for characterizing the performance and durability of ABS systems for commercialization. On the other hand, in industrial applications, the test systems is for evaluating the performance and durability of major ABS sub-systems. However, to the best of our knowledge, they can only perform very standard quality inspection tests and are lack the capability of automation and cannot diagnosis the possible cause of failure for improving system design.

Based on the observation mentioned above, a new versatile ABS testing system is required to perform the characterization of whole ABS system with more capability than the current existed ones. Therefore, in this paper, we propose and realize a new system based on industrial needs but also integrate the spirit of academic design on novel 
actuating, automation, and even on strengthen its capability for analyzing failures. A primary testing system has been realized in our previous research ${ }^{(1)}$. Similar to industrial available system, this design can perform performance and durability tests of $\mathrm{ABS}$ for industrial quality inspection purpose but with a novel design to triggering $\mathrm{ABS}$ via electronical signals only. In addition, this system is fully automatic with more embedded sensors for detecting temperatures and vibrations, which are both key information for failure analysis. With this system as the basis, the first goal of this paper is to further enhance the functionality of this test bench. Furthermore, a dynamic test bench will also be realized and integrated into the original test systems to evaluate the performance of ABS in real braking actions in the future. The enhanced original test bench will be named as the module test bench in this paper thereafter because the tested targets are focused on whole ABS or the HCU module. This system also has completely automatic operation and data analysis functions and can also be used for HCU. On the other hand, because it equip with rotated flywheel, the new dynamic test bench not only can be used to characterize the real braking performance of ABS modules but also served as a validating platform for evaluating the effectiveness of $\mathrm{ABS}$ control schemes in the future. Currently, our immediate goal is to use the whole system (i.e., the module test bench plus the dynamic test bench) as a bridge between static module performance tests and expensive road tests and a vehicle database can be drawn from the test data results for future virtual computer simulation.

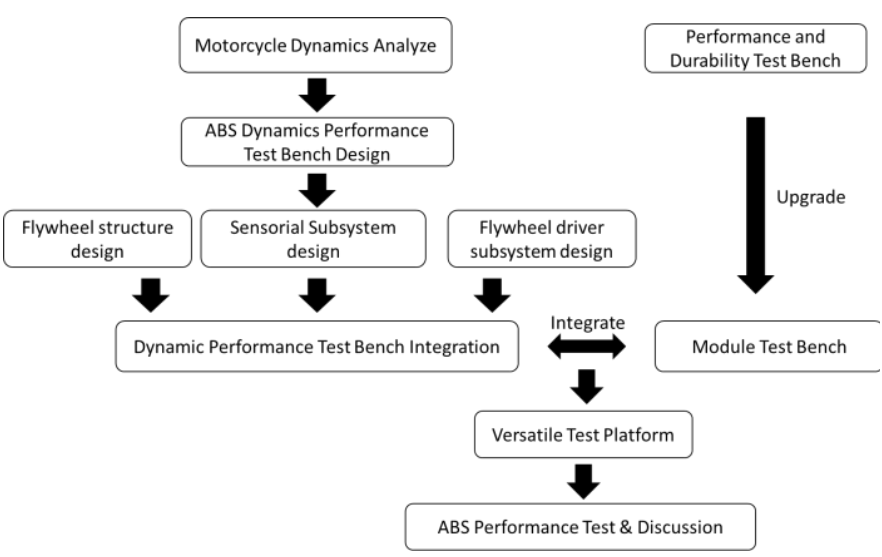

Fig. 1. Research flow.

The rest presents the technical detail and the research flow is shown in Fig. 1. In section 2, the ABS performance and durability test bench is introduced and the enhancement test made in this work is also explained. In section 3, the conceptual design of the ABS dynamic braking test bench is presented, including the motorcycle dynamics analysis. The important subsystems of the dynamic braking test bench are then presented in section 4, followed by the integration of these two test benches presented in section 5. The conclusion about the versatile ABS performance test bench we built are presented in section 6 .

\section{Realizing the module test bench}

\subsection{Introduction of ABS}

The typical ABS system operation flow is shown in Fig 2(a). While braking, the master cylinder squeezes the brake fluid and generates fluid pressure to the caliper. If the pressure to the caliper is too high that wheels are locked-up, ABS controller will close the inlet valve and open the release valve to reduce the brake fluid pressure for preventing locking. In general, an ABS consists of an ECU (electronic control unit), a $\mathrm{HCU}$ (hydraulic control unit) and a pump shown in Fig 2(b). The brake fluid released by the release valve is then pumped back to the master cylinder by pump.

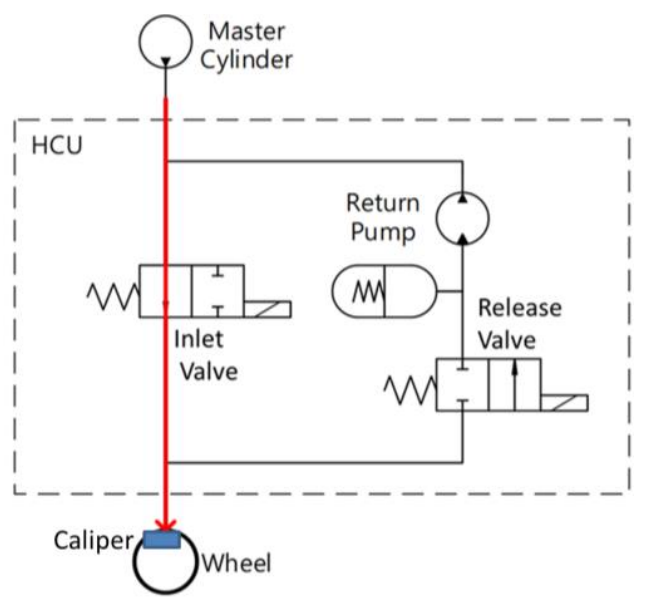

(a) Normal hydraulic brake operation

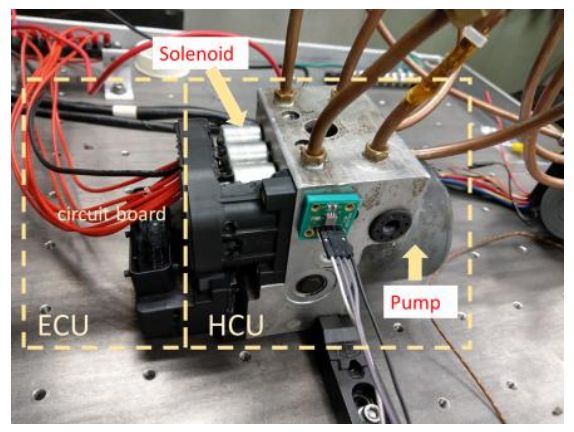

(b) ABS system components

Fig. 2. The fundamental ABS working principle. 


\subsection{Performance Test of ABS}

From the development perspective, the whole ABS, which consists of an ECU and an HCU, can be tested by the module test bench. However, technically, our collaborator may like to focus on developing their own HCU first. It is still possible to test HCU along by separating it from ECU and to attach external power and command mimicking by our electronic triggering system. By this approach, we can control each valve on and off independently. Detail setup can be found elsewhere ${ }^{(1)}$. With the mounted pressure sensors, the response and performance of HCU can be monitored. In addition, The test bench is also installed the current sensor and thermal couple for detecting the pump current and the brake fluid temperature for possible failure diagnostics. A human-machine interface (HMI) is also designed to conduct the six performance test and to calculate the corresponding performance index automatically. As shown in Fig. 3(a). The buttons on the panel provide different module test procedure for user to choose. The program could also, filters out noise, analyzes the obtained data and in-situ displays the test results., shown typically in Fig. 3(b) for determining the final performance.

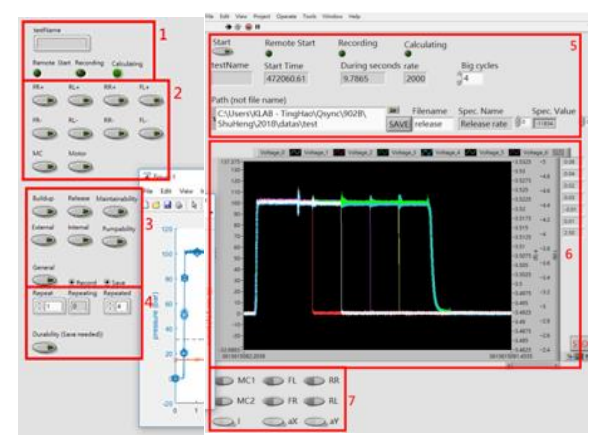

(a) The HMI of ABS module test bench

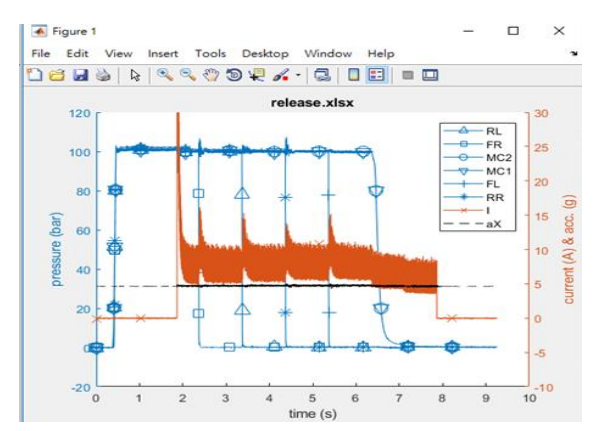

(b) Real-time analysis made by Matlab

Fig. 3. The automated test bench
Some ABS testing results are shown in Fig. 4. Fig. 4(a) shows the pump performance test result. The pumping efficient of the pump can be calculated. As shown in Fig. 4(b). This test basically reveals the bandwidth of pressure release and is directly linked to the response of brake in action. Since motor current, fluid temperature, and structure vibration are also monitored, it is possible to analyze these data for finding the major cause in case the HCU test fails to achieve satisfactory results.

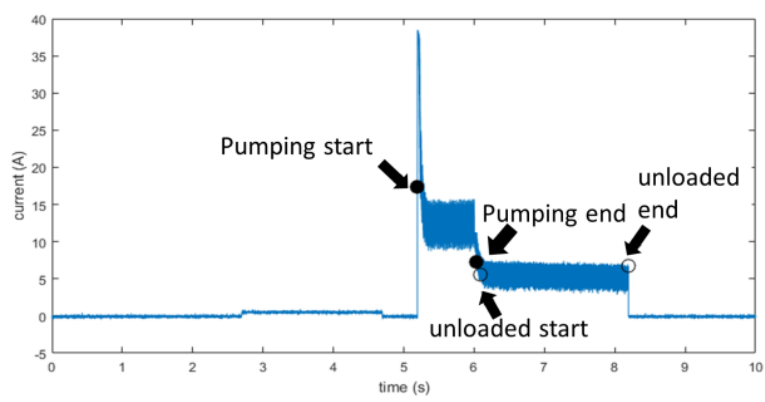

(a) The pump performance test

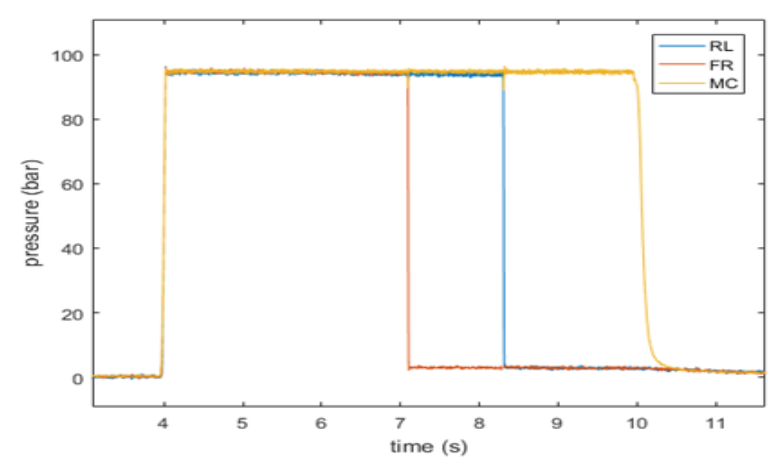

(b) The release valve performance test

Fig. 4. The experiment result of ABS module test bench

\subsection{Durability Test of ABS}

For demonstrating the capability of performing durability test, Bosch 9 is used as the test module. By sending a virtual speed signals from the function generator into the ABS to mimic the braking condition, the Bosch 9 is continuously operated. The temperature of working fluid is monitored by the attached thermal couples. As shown in Fig. 5, it can be seen that the fluid temperature increases continuously. Since the bulk modulus of hydraulic fluid varies with temperature, it implies that the braking force and bandwidth may also vary. In addition, the cyclic loading would also bring fatigue concern on ABS. It is expected that the performance degradation could be access by the pressure sensors and the corresponding longevity 
issues can also be reflected by the extra sensors we mounted in this test bench.

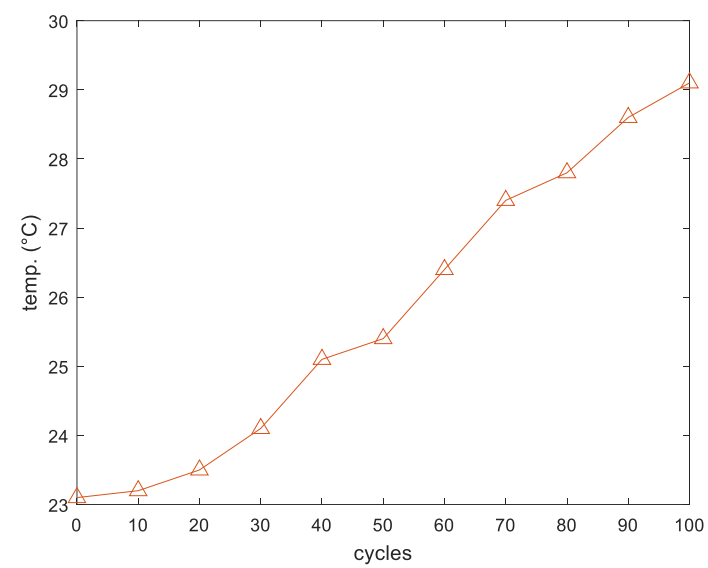

Fig. 5. The temperature changes in ABS durability test

\subsection{Virtual Wheel Speed Signals Test}

As addressed previously, electronic signals are used to create a virtual braking signal to trigger the Bosch 9 ABS. The signal generator is schematically shown in Fig. 6. The two-channel arbitrary signal generator export the virtual speed signals to the ABS. The virtual speeds rapidly increase in the first 10 seconds and dramatically decrease in the final 1 second. This significant deceleration is interpolated as the wheels lock up and the ABS will be triggered. This method not only can help us trigger ABS repeatedly without installing real wheels but also can help us analyze the ABS control logic. As shown in Fig 7. From the pressure fluctuate we know that although the wheels seem to lock up, ABS still doesn't completely release the brake, the threshold braking is still the main spirit of the anti-lock braking systems. And the pressure in the master cylinder become bigger than before, it's properly because the motor in the ABS pump pumped the released brake fluid back to the master cylinder. We can also find the big different between the brake fluid pressure sent to the front and rear wheels. It is properly because the normal force exerted from the road surface to the two wheels are different, so ABS were designed control two wheels in different logic.

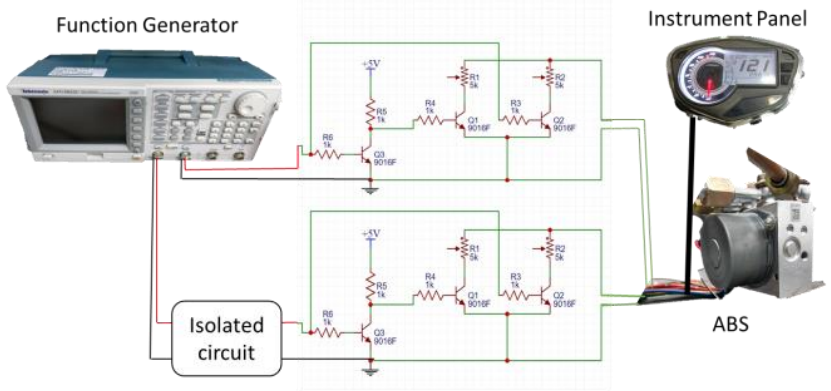

Fig. 6. The scheme plot of the virtual wheel speed generator

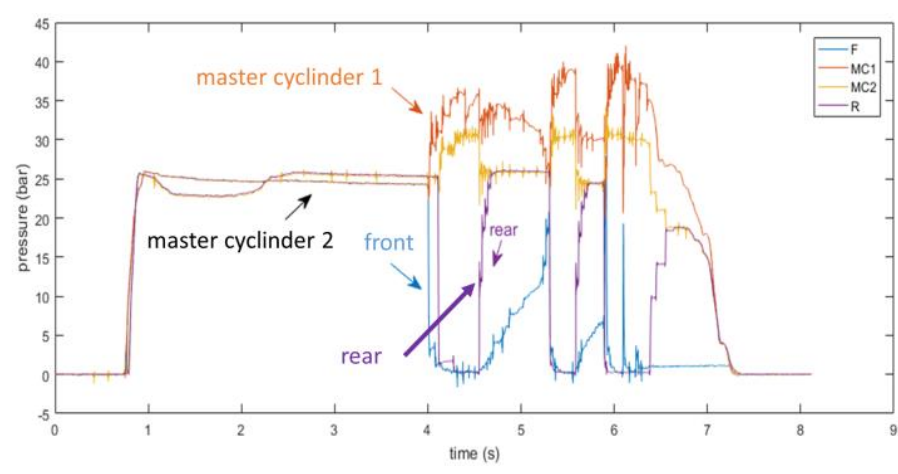

Fig. 7. The brake fluid pressure change when Bosch 9 actuating

\section{ABS Dynamic Test System Design}

\subsection{Motorcycle Dynamics Analysis}

For building a test environment which is similar to the motorcycle braking, we need to analyze the motorcycle dynamics first. When motorcyclist press the brake lever, the brake fluid pressure will transmit from the brake lever to the caliper and the braking disk will be squeezed. At this time, the wheel speed and vehicle speed become unsynchronized. To describe the phenomenon, slip formula has to be introduced.

$$
\lambda=\frac{\omega r-v}{v}
$$

$\lambda$ is the slip value, $\omega$ is the wheel angular speed, $r$ is the radius of the wheel, and $\mathrm{v}$ is the vehicle speed. Based on equation 1, we know that when the wheel is locked up and the vehicle is still moving forward, the slip will be 1 and the vehicle will be slipping. While the motorcycle is moving normally, the slip will be zero.

According to the previous study (3), the friction coefficient between the tire and the road depends on road 
surface and the slip (Fig. 8). The different slip values correspond to different friction coefficients, and the design goal of ABS is to control the slip so that the tire would have the greatest friction coefficient with the road surface and the vehicle could also maintain its steering ability. However, the real vehicle speed is difficult to obtain, so most ABSs are likely to calculate the slip by the relative speed of the front and rear wheels. Additionally, the friction force is not only the function of friction coefficient but also the function of normal force. Therefore, it is necessary to analyze the overall deceleration of the motorcycle and the normal force exerted by the road on the wheels.

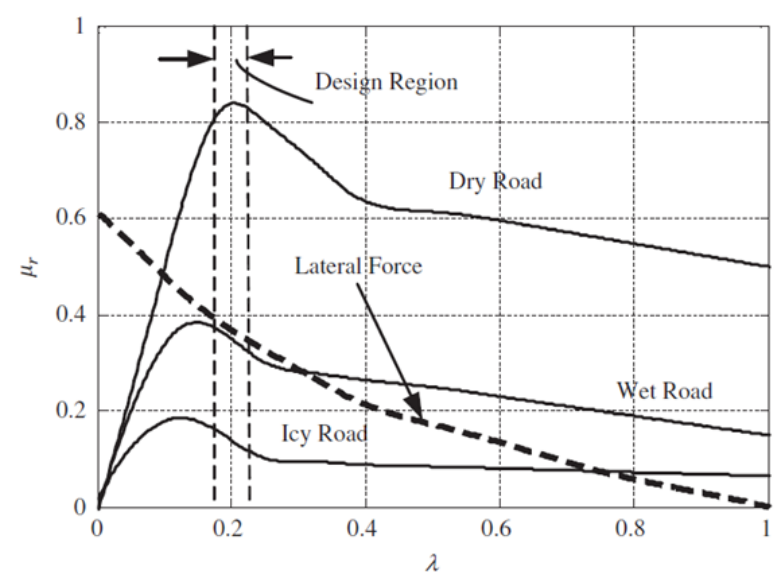

Fig. 8. The friction coefficient between the road and tire ${ }^{(3)}$

Take Motorcycle Dynamics ${ }^{(5)}$ as reference, the mechanical analysis of the motorcycle is shown in Fig. 9. This model does not consider structural deformation, wind resistance, and suspension. $m$ is the center of gravity of the motorcycle and the motorcyclist. The normal force exerted by the road on the wheels is $\mathrm{Nf}$ and $\mathrm{Nr}$, the frictional force exerted on the wheels is Ff and Fr, and $\ddot{x}$ is the acceleration of the vehicle. The force balance equations are listed in equation (2) and (3), and the moment balance equation is listed in equation (4). After the variable is substituted, the solution of the equations is plotted in Fig. 10. The normal force acting on the front and rear wheels are different during braking, which means that the distribution of the braking forces is also different. Therefore, it is an opportunity to calculate the slip by detecting the speed of the front and rear wheel. The Fig. 10 shows that the deceleration is proportional to the friction coefficient. The trend of the deceleration change is important because it corresponds to the braking distance directly. To sum up, the goal of the dynamic braking test bench is to imitate the deceleration and the normal force change while motorcycles are during braking. If the test bench with these dynamic characteristics can be realized, the ABS performance statistics collected from test bench will approach to the field test. It is helpful for us in developing ABS control strategy or analyzing the commercial ABS on the test bench.

$$
\begin{aligned}
& m g=N f+N r \\
& -N f \mu_{f}-N r \mu_{r}=m \ddot{x} \\
& N f(p-b)=b N r+F f h+F r h
\end{aligned}
$$

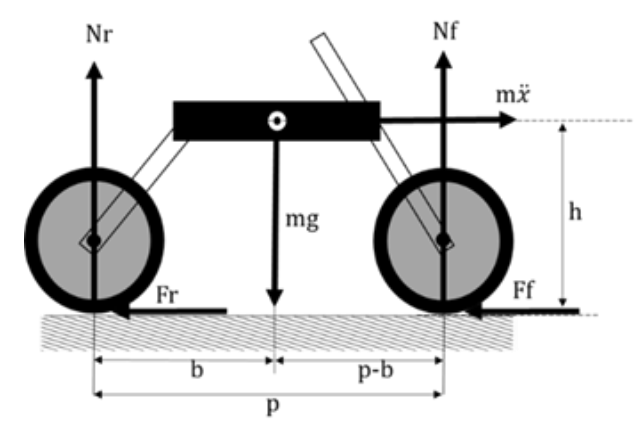

Fig. 9. Free body diagram of the motorcycle

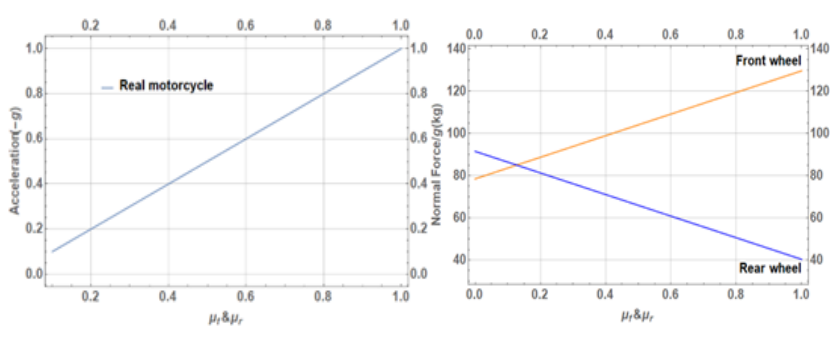

Fig. 10. Motorcycle dynamics analysis

\subsection{Dynamic Test Bench Conceptual Design}

Based on the analysis of motorcycle braking dynamics in this study and the relevant literature, we conclude that two test bench designs may reflect the dynamic characteristics of motorcycle braking. The first one uses the scaled-down model to analyze the real vehicle braking distance ${ }^{(4)}$. However, it is extremely difficult to develop a scaled-down motorcycle model with a complete braking system. The other one uses an actuator to control the normal force between the flywheel and the tire. The difficulty and uncertainty of this method are actuator control and installation ${ }^{(2)}$. In order to have a simpler and more effective test bench design, this study chose the flywheel design which is often used by the academia as a source of kinetic energy. However, we proposed a new architecture to provide enough normal force between the 
flywheel and wheels. The structural design is simple and remains the braking dynamics characteristics such as the normal force distribution.

This design is shown in Fig. 11. The flywheel is placed above the two wheels for rotation. This situation simulates the motorcycle running on the road. The angles $\theta$ and $\alpha$ between the front wheel, rear wheels and the flywheel can be adjusted according to the distribution of the center of gravity of various models. The moment inertia of the flywheel is adjusted for the different weight of the motorcycle. The normal force of the front and rear wheels of the motorcycle is adjusted by the ratio of 12 and 13 . From our calculation, the unconstrained flywheel can achieve the center of gravity shifting while braking. However, a no restriction flywheel may cause many problems, so we proposed a properly restrained method to prevent the flywheel system unstable. The spring on the left side not only stabilizes the rotation performance of the flywheel but also prevents flywheel from bouncing. Besides, springs can also provide a pre-force to help the flywheel and tire contact normal force adjustment.

The dynamic performance of the flywheel system is shown in Fig. 12. After we tuned the parameter of the flywheel system, the normal force change is shown in Fig. 12(a). Although the dynamics performance seems different to the motorcycle, the Fig .12(b) shows that the ratio of the normal forces exerted on the wheels nearly the same. From the slip control opinions and the ABS control strategy we guess in section 2.4, having the correct normal force distribution ratio is enough for the development of ABS on the test bench. As shown in Fig. 12(c). Compare to the motorcycle braking deceleration we calculate before, the trend of these two system are the same in some particular friction coefficients. Although it is impossible to make two curves totally the same, according to the friction coefficient of the flywheel, we still can make the curve nearly the same in particular friction coefficient section.

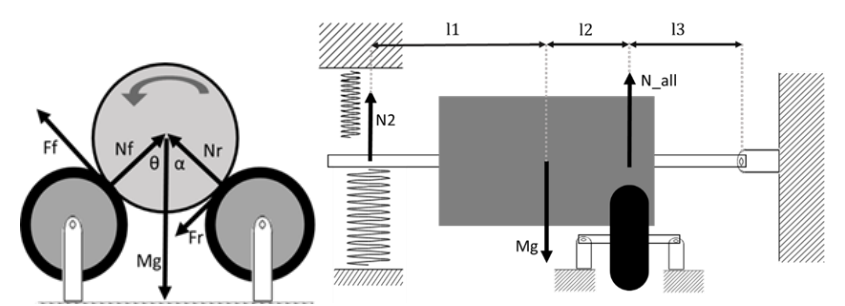

Fig. 11. The conceptual design of the dynamic braking test bench

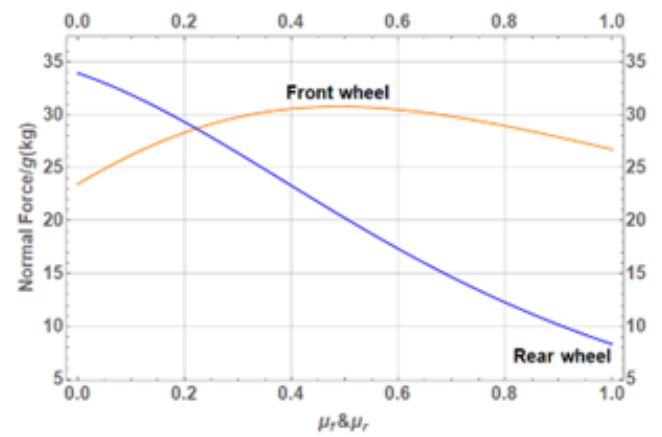

(a) Normal force translation

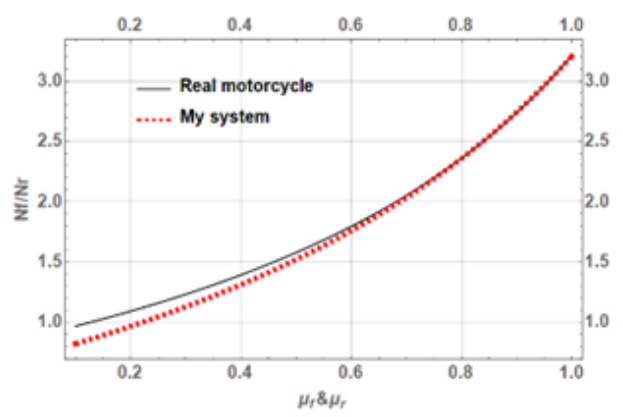

(b) Normal force distribution ratio

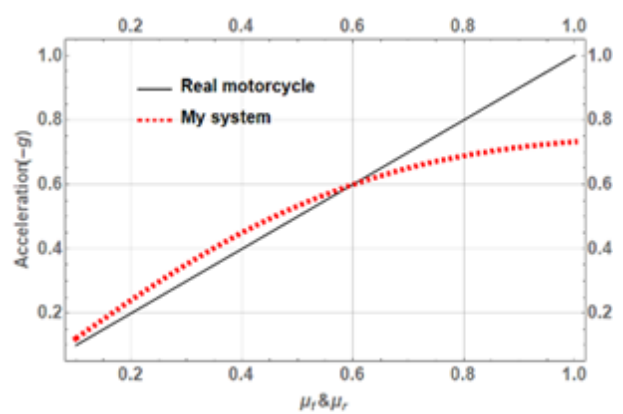

(c) Acceleration change through different slip

Fig. 12. The analysis of the dynamic braking test bench

\section{ABS Dynamic Test Bench Components Design}

\subsection{Flywheel Structure Design}

The design of the ABS test bench is shown in Fig.13. The flywheel is the key part of the whole system, which is made of structural steel. It is difficult to set up the flywheel with the same kinetic energy corresponding to the motorcycle. Besides, the kinetic energy of the motorcycle is not a major factor affecting ABS control. Therefore, after discussing the required strength and speed of the drum, and the difficulty during the manufacturing process with the manufacturer, the flywheel was designed. The flywheel should be able to achieve a dynamic balance of $1000 \mathrm{rpm}$ 
which is corresponding to the motorcycle running at a speed of $90 \mathrm{~km} / \mathrm{hr}$. The flywheel is supported by two screw jacks so that it will be easy to adjust the height of the flywheel. Moreover, the turning and tilting behaviors of motorcycles are also achievable.

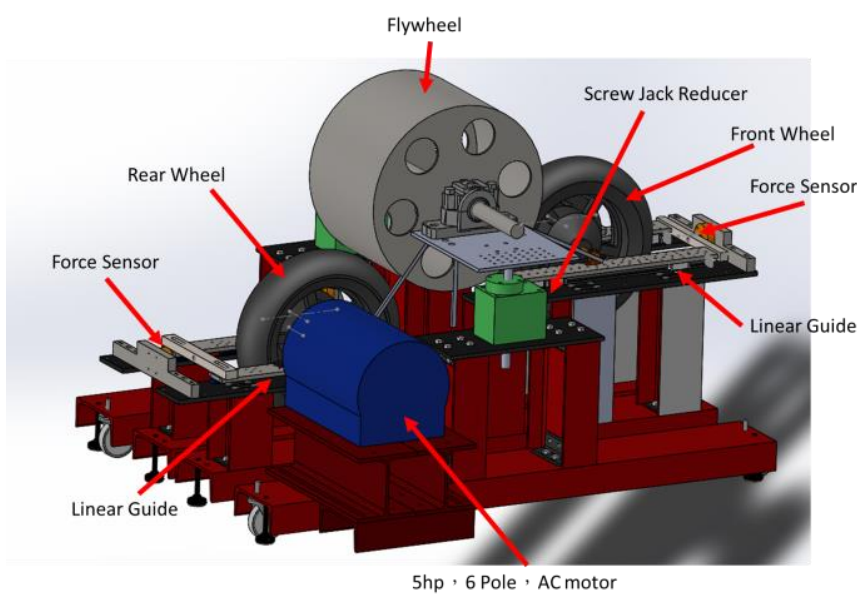

Fig. 13. The sketch plot of the dynamic braking test bench

\subsection{Flywheel Driver Subsystem Design}

To drive the flywheel, the rear wheel of the motorcycle is connected with the motor and then transmits the rotational kinetic energy to the entire system. In order to make the system brake without excessive resistance interference, the Fig.14 shows the small clutch is designed to completely separate the motor from the system after the acceleration is completed without interfering the load cell measurement. By making the special shape on the end of the transmission shaft, the clutch can smoothly and completely the shaft matching.

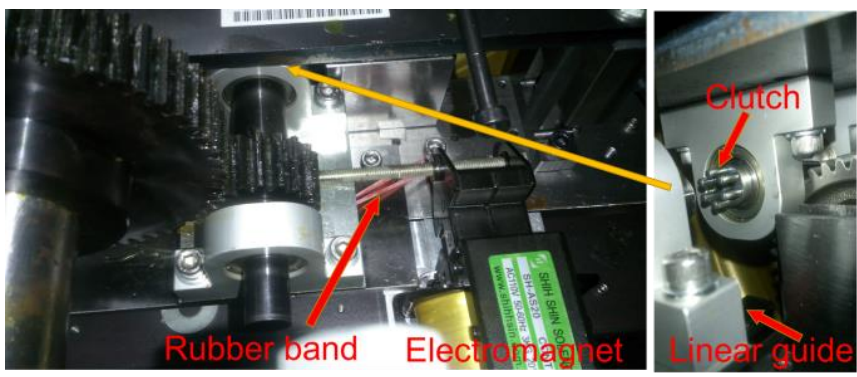

Fig. 14. The placement of the load cell in the test bench

\subsection{Sensorial Subsystem Design}

Based on the existing brake fluid circuit sensor sub-system of the module test system, the brake dynamic performance test system focus on detecting the braking force and the normal force. In this study, pancake load cell is used as the sensor. As shown in Fig. 15. The load cell with the linear guide can measure the force in a particular direction. The friction force between the caliper and the braking disk is first measured by the load cell. By using rotation rigid body dynamics, we can acquire the braking force between the wheel and the flywheel. The other load cell can measure the horizontal force of the normal force which flywheel exert on the wheel. In addition, the Hall sensor and the sensor rings on the wheels is used to measure the front and rear wheel speeds, and the encoder is used to measure the flywheel speed. As a result, important parameters of the brake can be obtained.

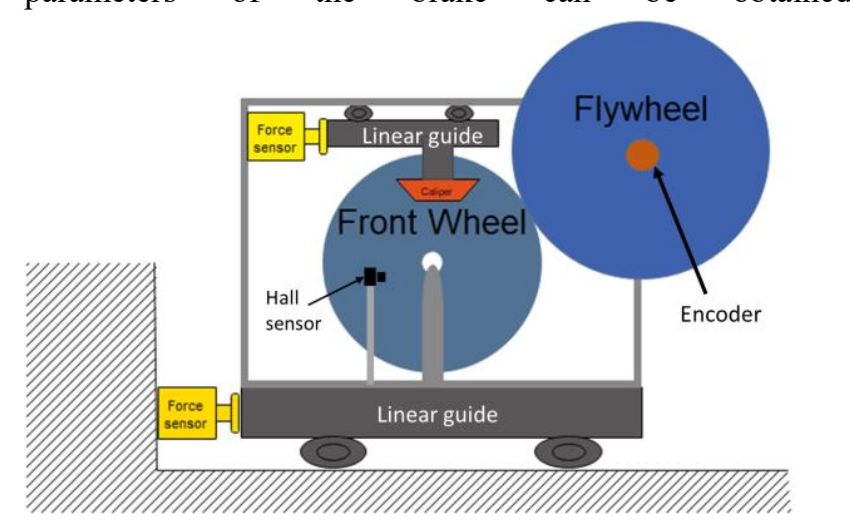

Fig. 15. The placement of the load cell in the test bench

\section{System Integration}

\section{1 dynamic test system assembly}

The flywheel is supported by the bearing house and two wheels (Fig. 16). The combination of linear guide and springs at the end of the shaft is a special design for preserving the flywheel applying different force to the wheels in different slip situation. There are two linear guides under each caliper, which is installed on the steel structure attached to the load cell. There are four linear guides support two wheels and are fixed with the other two load cells.

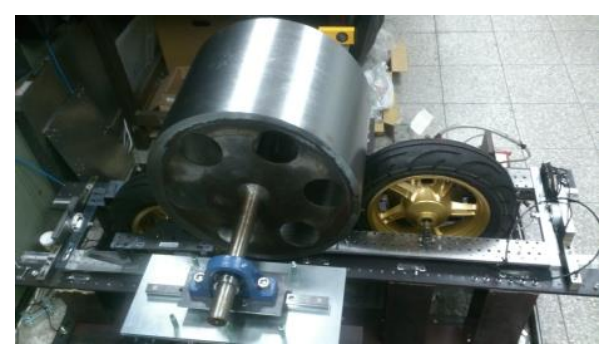

Fig. 16. Dynamic braking performance test bench 


\subsection{Module test bench and Dynamic test bench integration}

Two systems are placed together and used to build the new manifold blocks for connecting the brake fluid pipe to the caliper of the dynamics test bench (Fig. 17). In the new manifold block, we also installed the temperature sensor which can be buried into the brake fluid. It means we can measure the brake fluid temperature of the brake fluid directly. The stainless cylinder is added for adjusting the volume of the brake fluid. In this way, the fluid system will more flexible to simulate any size of a vehicle.

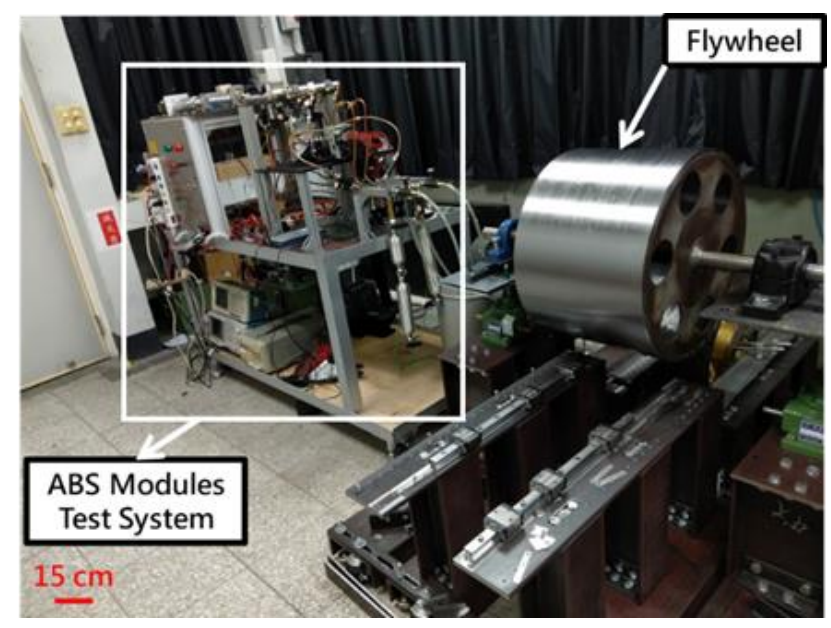

Fig. 17 . The versatile test bench

\section{Conclusion}

This paper presents the design of a novel ABS braking performance test bench based on the enhanced module test bench, by which some ABS tests were conducted. The complete series of HCU performance test done on the test bench validated the ability of the test bench. In the durability test, the relation between the temperature and the ABS working cycles was found. The Bosh 9 control strategy was analyzed using pressure sensors and the virtual wheel speed signals. Additionally, the new dynamic braking performance test bench was designed according to the simplified motorcycle braking dynamics. After estimating the dynamic characteristics of the test bench, the system was realized by integrating three parts: flywheel structure, flywheel driving subsystem, and sensorial subsystem.

In the future, by installing Bosch 9 ABS to the flywheel system, we can validate the feasibility of the test bench. After the Bosch 9, ABS braking performance is confirmed on the test bench, the statistics collected from the test bench should be compared to the statistics collected from the field test. Although the characteristics of the braking performance we collected from the test bench and real motorcycle would be different, some correlation between these two systems could still be found.

\section{Acknowledgment}

This work is supported by Ministry of Science and Technology (MOST) of Taiwan under contract numbers 105-2221-E-006-100-MY3, and by the Yen Tjing Ling Industrial Development Foundation. The comments and assistances from Prof. S.-F. Ling and Mr. C.-E. Wu from Lioho Machine Works, Ltd are great appreciated.

\section{References}

(1) C. H. Wu, S. H. Guo, and K. S. Chen "Design and Realization of a Novel Performance and Durability Test Platform for Anti-lock Braking Module", 6th IIAE International Conference on Industrial Application Engineering, Mar. 2018, Okinawa, Japan.

(2) M. C. Wu and M. C. Shih : "Simulated and experimental study of hydraulic anti-lock braking system using sliding-mode PWM control", Mechatronics, Vol. 13, No. 4, pp. 331-351, 2003

(3) C. V. Altrock: "Fuzzy logic technologies in automotive engineering", in WESCON/94. Idea/Microelectronics. Conference Record, Anaheim, CA, USA, 1994.

(4) R. G. Longoria, A. Al-Sharif, C. B. Patil: "Scaled vehicle system dynamics and control:a case study in anti-lock braking”, Int. J. Vehicle Autonomous Systems, Vol. 2, Nos. 1/2, 2004.

(5) V. Cossalter, Motorcycle Dynamics , 2th ed., Race Dynamics, 2002 